\title{
Forschung in der Industrie: \\ Möglichkeiten und Grenzen \\ staatlicher Steuerbarkeit
}

\section{Edgar Grande und Jürgen Häusler}

$92 / 3$

Max-Planck-Institut für Gesellschaftsforschung

MPIFG Discussion Paper $92 / 3$

ISSN $0933-5668$

Lothringer Str. 78

März 1992

D-5000 Köln 1

Marrz 1992

Federal Republic of Germany

(RFC822): mpi@mpi-fg-koeln.mpg.dbp.de

(X400): $\quad \mathrm{S}=\mathrm{mpi} ; \mathrm{O}=$ mpi-fg-koeln; $\mathrm{P}=\mathrm{mpg} ; \mathrm{A}=\mathrm{dbp} ; \mathrm{C}=\mathrm{de}$

(PSI): \begin{abstract}
PSI\%4505011006::MPI
\end{abstract}
Telephone

Fax

0221 / 33605-0 


\begin{abstract}
The lion's share of West Germany's R\&D activities is conducted in industrial $R \& D$ labs funded by industry. Nevertheless, industrial R\&D has been largely neglected by political scientists as a topic for empirical research. This study aims at bridging this gap. It concludes that the opportunity for government to control industrial R\&D can neither be unconditionally assumed nor simply denied. Given the complexity and variability of the internal structures of industrial R\&D, it appears feasible that government officials may indeed selectively intervene in $R \& D$ related decision-making processes in industry.
\end{abstract}

Der weitaus größte Teil der Forschungs- und Entwicklungsarbeiten in der Bundesrepublik wird in der Wirtschaft durchgeführt und von dieser finanziert. Dennoch hat die Industrieforschung in politikwissenschaftlichen Analysen zur staatlichen Techniksteuerung bislang keine Beachtung gefunden. Die vorliegende Studie beabsichtigt, diese Forschungslücke zu schließen. Sie kommt zu dem Ergebnis, daß die externe Steuerbarkeit der Industrieforschung weder umstandslos vorausgesetzt, noch einfach bestritten werden kann. Die Komplexität und Variabilität der unternehmensinternen FuE-Strukturen eröffnet staatlichen Akteuren durchaus die Möglichkeit der gezielten Intervention und der selektiven Partizipation.

\title{
9411261 \\ Max-Planck-Institut \\ für Gesellschaftsforschung \\ Bibliothek \\ $P(A-7 / 9203$ \\ 28032000
}




\section{Inhalt}

Abstract 2

1. Einleitung 5

2. Zur Bedeutung der Industrieforschung 7

3. Ambivalenz der Industrieforschung 10

4. Steuerungsprobleme des industriellen Forschungsmanagements 14

5. Chancen und Grenzen staatlicher Techniksteuerung in der Industrieforschung

6. Forschungsperspektiven 24

Literatur 26 


\section{Einleitung}

Der weitaus größte Teil der Forschungs- und Entwicklungsarbeiten in der Bundesrepublik wird in Unternehmen der Wirtschaft durchgeführt und von diesen finanziert. Nicht an Hochschulen oder in staatlichen Forschungseinrichtungen, sondern in den Forschungs- und Entwicklungseinrichtungen der Unternehmen wird der Großteil der technologischen Fakten generiert und "gehärtet", werden die technologischen "Tatsachen" geschaffen. Für eine politische Techniksteuerung, die "vor Vollendung der Tatsachen" (Hack 1988) ansetzen will, müßte folglich die Industrieforschung das zentrale Steuerungsobjekt sein.

Um so mehr erstaunt es, daß die Industrieforschung bislang in politikwissenschaftlichen Analysen zur staatlichen Techniksteuerung keine Beachtung gefunden hat. Weitgehend "politik"- und "staatszentriert", widmet sich die politikwissenschaftliche Literatur zur Forschungs- und Technologiepolitik, soweit sie nicht nur ideologiekritisch gemeint ist (vgl. u.a. Narr/ Offe 1976, Ronge 1977), vorrangig der Beschreibung und Analyse der staatlichen Technologiepolitik ("policy"). Gefragt wird nach der relativen Bedeutung staatlicher Ausgaben für Forschung und Entwicklung (Väth 1984, Lehner 1986, Fuhrmann/ Väth 1990), den programmatischen Etappen der staatlichen Technologiepolitik sowie ihres sich wandelnden Instrumentariums (Bruder/ Dose 1986; Wollmann 1989), oder auf institutioneller Ebene nach der Herausbildung und Verteilung forschungspolitischer Kompetenzen (Stucke 1991). Dagegen ging z.B. kein Beitrag des DVPW-Kongresses zum Leitthema "Politik und die Macht der Technik" explizit auf Strukturen und Entwicklungslinien der Industrieforschung ein (vgl. Hartwich 1986); ein ähnliches Bild ergibt sich beim Blick in einschlägige Beiträge zu politikwissenschaftlichen Wörterbüchern (vgl. Bräunling 1983) und Sammelbänden (vgl. Bruder 1986) sowie in Monographien zur FuT-Politik in der Bundesrepublik (von Alemann et al. 1988). Aus politologischer Sicht muß daher bezüglich der Struktur und Funktionsweise unternehmerischer Forschungs- und Entwicklungsaktivitäten ein massives Forschungsdefizit konstatiert werden: "Wie diese 'private' Industriepolitik der Großkonzerne funktioniert, welche Institutionen und Mechanismen der Kooperation und Konfliktregulierung hier existieren, ist sozialwissenschaftlich bisher kaum erforscht" (Esser 1990: 62-63; vgl. auch Esser 1987 und Simonis 1989). Noch immer gilt also zumindest für den forschungspolitischen Diskurs die schon 1962 geäußerte Kritik: "Except as it enters as a participant in the 
general political arena, the business firm has not been a focus of study for political scientists" (March 1988: 101). ${ }^{1}$

Diese Vernachlässigung des für die FuT-Politik zentralen Steuerungsobjektes mag im professionellen Selbstverständnis der Disziplin angelegt sein. Will man aber Aussagen zu staatlichen Steuerungspotentialen machen, so wird eine solche Beschränkung problematisch. Denn dann genügt es nicht, sich der staatlichen Steuerungsfähigkeit zu vergewissern, erforderlich sind auch Kenntnisse über ihr Steuerungsobjekt. Empirische Analysen staatlicher Steuerungspotentiale können sich nicht auf den Bereich staatlicher Politiken beschränken, denn das staatliche Steuerungspotential ergibt sich nicht allein aus der Summe der dem Staat zur Verfügung stehenden Kompetenzen und Ressourcen. Es ist vielmehr das Produkt von zumindest drei kritischen Strukturvariablen: (i) der staatlichen Binnenstruktur und der daraus resultierenden staatlichen Steuerungsfähigkeit; diese Strukturvariable bemißt die institutionellen Kapazitäten, die Ausstattung mit Steuerungsinstrumenten und das Ausmaß strategischer Kohärenz auf staatlicher Seite; (ii) der Binnenstruktur des zu steuernden Steuerungsobjekts und seiner daraus resultierenden Steuerbarkeit; mit der spezifischen Integration und Organisation der FuE-Aktivitäten im Unternehmenszusammenhang werden die strukturellen Opportunitäten und Restriktionen gesetzt, auf die staatliche Politik in ihrem Versuch der Steuerung industrieller Forschungsbemühungen trifft; (iii) den interorganisatorischen Beziehungen und Interaktionslogiken zwischen dem Staat und dem zu steuernden gesellschaftlichen Teilsystem; hiermit wird insbesondere der Grad der informationellen Vernetzung und damit nicht zuletzt das Ausmaß staatlicher Empathie gegenüber den Entscheidungssituationen und -kalkülen des Unternehmenssektors bemessen.

Die Erfolgsaussichten einer staatlichen Steuerung von Industrieforschung sind folglich mehrfach kontingent. Sie sind zunächst zweifellos abhängig von der Fähigkeit des Staates, Ressourcen strategisch zielgerichtet bereitzustellen und einzusetzen. Ohne ausreichende institutionelle Kapazitäten, ohne geeignete Steuerungsinstrumente und ohne ein Mindestmaß an strategischer Kohärenz müßte der Staat auch noch die günstigsten Steuerungschancen ungenutzt lassen. Damit alleine ist es aber nicht getan. Auch ein üppig ausgestattetes und wohl administriertes staatliches Förderprogramm würde ohne signifikante Folgen bleiben, wenn es bei den Unternehmen keine Resonanz finden oder

1 Wichtige Arbeiten zur Industrieforschung - wenn auch nicht mit steuerungstheoretischem Fokus - liefern dagegen die Soziologie (vgl. z.B. Hack 1988, 1990, Rammert 1988, Zündorf/. Grunt 1982) und die Betriebswirtschaft (vgl. z.B. Biegel 1987, Bleicher 1990, Brockhoff 1988, de Pay 1989, Rotering 1990, Zweipfennig 1991). 
lediglich bereits laufende und intern finanzierte Forschungs- und Entwicklungsarbeiten honorieren würde. Der Erfolg staatlicher Techniksteuerung ist folglich immer auch abhängig von den strukturellen Opportunitäten und Restriktionen, die die Unternehmen der Politik bieten. Diese dürfen nicht mit den abstrakten "Verwertungs"- und "Zahlungs"-Logiken der Ökonomie gleichgesetzt werden. Für die Steuerbarkeit der Industrieforschung wichtiger sind die konkreten Wettbewerbsbedingungen der Unternehmen, ihre Binnenstrukturen, Präferenzen und Strategien, sowie die spezifischen Formen der Integration und Organisation von Forschungs- und Entwicklungsaktivitäten in den Unternehmen. Diesbezügliche Kenntnisse liegen in der politikwissenschaftlichen Literatur bisher jedoch nicht vor.

Auf die Schließung dieser Forschungslücke zielt der vorliegende Beitrag². Beabsichtigt ist, (1) einen Überblick über die quantitative Bedeutung der Industrieforschung in der Bundesrepublik zu bieten, (2) die strukturellen Dilemmata von Forschung in Wirtschaftsunternehmen herauszuarbeiten, um daraus (3) die unternehmensinternen Steuerungsnotwendigkeiten und -probleme sowie (4) die externen Steuerungschancen und -grenzen für den Staat zu bestimmen.

\section{Zur Bedeutung der Industrieforschung}

Bereits ein erster Blick auf die Struktur der bundesdeutschen Forschungslandschaft offenbart den herausgehobenen Stellenwert der Forschungs- und Entwicklungsarbeiten, die in Einrichtungen der Industrie stattfinden. Der weitaus größte Teil der FuE-Aktivitäten in der Bundesrepublik wird in Unternehmen der Wirtschaft durchgeführt und von diesen auch finanziert. Von den gesamten FuE-Aufwendungen, die 1990 etwas über $70 \mathrm{Mrd}$. DM betrugen, finanziert der Wirtschaftssektor knapp 64\%, an der Durchführung von FuE ist die Industrie gar mit über $70 \%$ beteiligt. An Hochschulen dagegen werden lediglich etwa $14 \%$ der FuE-Aktivitäten durchgeführt und in staatlich finanzierten außeruniversitären Forschungseinrichtungen (Max-Planck-Gesellschaft, Fraunhofer-Gesellschaft, Großforschungseinrichtungen, Instituten der Ressortforschung u.a.) etwa 13\% (BMFT 1990: 340-341).

2 Der Beitrag referiert Teilergebnisse eines von den Autoren am Max-PlanckInstitut für Gesellschaftsforschung durchgeführten Forschungsprojekts zum Thema "Industrieforschung und staatliche Politik". Im Rahmen des Projekts wurden zahlreiche Interviews mit Experten in Unternehmen, Verbänden, Forschungseinrichtungen und staatlichen Stellen durchgeführt, auf die sich der folgende Beitrag wiederholt bezieht, ohne daß hierauf jeweils explizit hingewiesen wird. 
In historischer Perspektive fällt auf, daß trotz expandierender staatlicher Forschungshaushalte, des Aufbaus institutioneller Kapazitäten im Bereich staatlicher Forschungsförderung, der ständigen Ausweitung staatlich geförderter Technologiebereiche, der Erweiterung des dabei genutzten Instrumentariums und des Auf- und Ausbaus staatlicher Forschungseinrichtungen der Staatsanteil am Forschungssektor nicht zugenommen, sondern abgenommen hat. Entgegen der noch in den 60er Jahren verbreiteten Erwartung (vgl. Weinberg 1970) fand in der Bundesrepublik keine "Verstaatlichung" der Forschung statt, sondern das Gegenteil: ihre allmähliche Privatisierung. Der Anteil des Staates bei der Durchführung von FuE ging, nach einem kurzen und leichten Anstieg gegen Ende der 60er Jahre, zwischen 1962 (40\%) und 1989 (26\%) kontinuierlich zurück. Ähnliches gilt für die Finanzierung von Forschung und Entwicklung: 1962 finanzierte der Staat noch etwa die Hälfte aller FuE-Aktivitäten, während sein Anteil 1990 nur noch 34\% betrug. Spiegelbildlich hat die relative - zumindest quantitative - Bedeutung des Wirtschaftssektors im Forschungssystem der Bundesrepublik insbesondere seit Mitte der 70er Jahre deutlich zugenommen; so erhöhte sich sein Anteil an der Durchführung von FuE zwischen Mitte der 70er und Mitte der 80er Jahre um mehr als zehn Prozentpunkte, sein Finanzierungsanteil stieg zwischen Mitte der 70er und Ende der 80er Jahre gar um 15 Prozentpunkte (vgl. Schaubild 1 sowie Häusler 1989 und BMFT 1990: 339).

Schaubild 1: FuE-Aktivitäten in der Bundesrepublik nach Sektoren

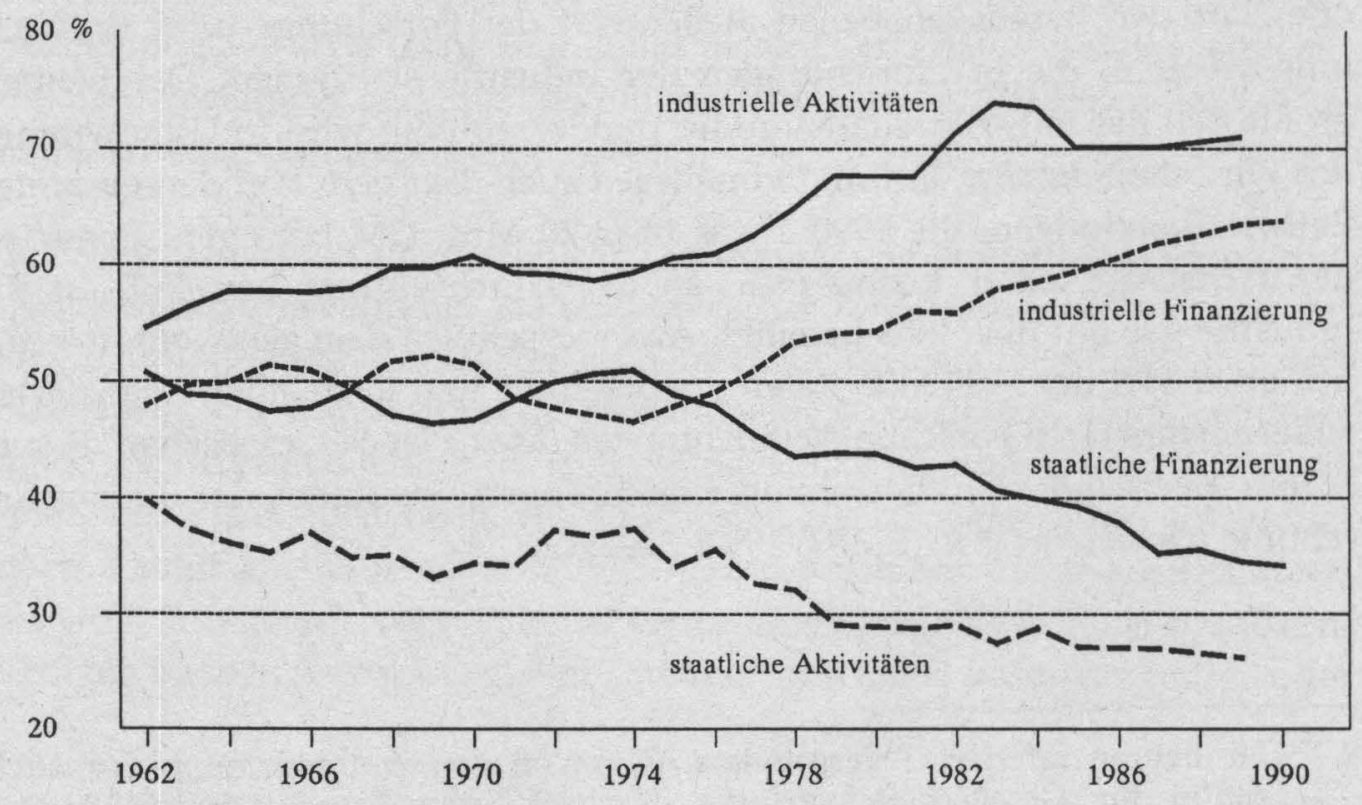


Eine vergleichbar zentrale Stellung des Wirtschaftssektors im jeweiligen nationalen Forschungs- und Innovationssystem findet sich im internationalen Vergleich nur in wenigen Industrieländern. Was den Finanzierungsanteil angeht, erreicht der private Sektor eine ähnliche quantitative Bedeutung nur noch in der Schweiz, in Belgien, in Japan und in Schweden (in dieser Ländergruppe liegen die Anteile bei 60-75\%). In den meisten OECD-Staaten finanziert die Industrie lediglich $40-50 \%$ der nationalen FuE-Aktivitäten; so liegt die industrielle Finanzierungsquote beispielsweise in den Niederlanden bei knapp über $50 \%$, in Großbritannien und in den USA bei etwa $50 \%$, und in Italien und Frankreich bei lediglich $45 \%$. Was die Durchführung von FuE angeht, lassen sich innerhalb der OECD drei Ländergruppen bilden: Von der Industrie durchgeführt werden etwas über $70 \%$ der nationalen FuE-Aktivitäten neben der Bundesrepublik auch in der Schweiz, in Belgien und in den USA; knapp unter 70\% beträgt der Anteil in Japan, in Großbritannien und in Schweden; unter $60 \%$ dagegen zum Beispiel in Frankreich und Italien (OECD 1991).

In zweifacher Hinsicht sind die industriellen FuE-Aktivitäten durch eine deutliche Konzentration gekennzeichnet. Zum einen finden fast drei Viertel der Aktivitäten in nur vier Branchen statt: Elektrotechnik (28\%), chemische Industrie (20\%), Automobilindustrie (15\%) und Maschinenbau (10\%). Zum anderen vereinen Unternehmen mit mehr als 10.000 Beschäftigten über die Hälfte (1987: 58\%) der FuE-Aktivitäten im Wirtschaftssektor auf sich; mit einem etwas geringeren Anteil an den in der Industrie Beschäftigten (51\%) und einem deutlich geringeren Anteil am Gesamtumsatz (46\%) bestreiten sie einen überproportionalen Teil der Industrieforschung (SV 1990: 52-53 und für Langzeittrends Häusler 1989).

Aktuellen Trends einer verstärkten externen Zusammenarbeit und Internationalisierung (vgl. etwa van Tulder/ Junne 1988) zum Trotz, findet der wesentliche Teil der FuE-Aktivitäten bundesdeutscher Unternehmen in den Unternehmen und im Inland statt. Der Anteil intern durchgeführter FuE liegt im Durchschnitt bei über 90\% (SV 1990: 50); der Anteil externer FuE hatte sich zwar zwischen $1969(3,6 \%)$ und $1983(9,8 \%)$ nahezu verdreifacht, verbleibt seither jedoch auf diesem Niveau oder verringert sich sogar wieder (1985: 9,0\%; 1987: 8,4\%) (Häusler 1989: 74 und SV 1990: 50). Auch Untersuchungen einzelner bundesdeutscher Unternehmen (Bullinger 1990) sowie internationaler Unternehmen (EIRMA 1989) beziffern den Anteil extern durchgeführter FuE im Unternehmenssektor auf etwa zehn Prozent. Studien ausgewählter Großkonzerne ergaben einen Anteil der FuE-bezogenen Auslandsaufwendungen von etwa $10 \%$ Jungnickel et al. 1977, Pausenberger 1982) und für im Aus- 
land beschäftigte FuE-Mitarbeiter durchschnittlich einen Anteil von unter $20 \%$ (Dörrenbächer/ Wortmann 1991). ${ }^{3}$

Nicht nur im Hinblick auf die zuletzt gemachten Angaben muß für den Bereich industrieller FuE auf massive Branchendifferenzen deutlich hingewiesen werden. So variiert der Anteil der im Ausland beschäftigten FuE-Mitarbeiter zwischen 10\% (Automobilbau), 20\% (Elektrotechnik) und fast 30\% (Chemische Industrie) (Dörrenbächer/ Wortmann 1991). Der Anteil extern durchgeführter FuE schwankt, je nach Quelle, zwischen etwa 2\% (Textilindustrie) und 8\% (Elektrotechnik) (SV 1990: 50) oder etwas über 10\% (Anlagen- und Maschinenbau, Elektrotechnik) und knapp 20\% (Automobilbau) (Bullinger 1990). Die FuE-Intensität (gemessen als Umsatzanteil der FuE-Aufwendungen) schwankt zwischen knapp über 3\% (Maschinenbau), um 5\% (Chemische Industrie, Feinmechanik und Optik), knapp unter $8 \%$ (Elektrotechnik) und fast 30\% (Luft- und Raumfahrt) (Häusler 1989: 67). Schließlich fließen staatliche FuE-Fördermittel selektiv in vor allem drei Branchen: Luft- und Raumfahrt (fast 30\% der Mittel) sowie Elektrotechnik und Maschinenbau (jeweils etwa 20\%); damit variiert die Eigenfinanzierungsquote zwischen einzelnen Branchen und reicht von $98 \%$ (Chemische Industrie) bis $\mathrm{zu} 32 \%$ (Luft- und Raumfahrt) (SV 1990: 48-49).

Zusammengefaßt verweist diese knappe Analyse der quantitativen Strukturen im bundesdeutschen Innovationssystem auf die bedeutende und in den letzten fünfzehn Jahren an Bedeutung gewinnende Stellung der Industrieforschung. Unter der Annahme, daß auch Teile der in sonstigen wissenschaftlichen Einrichtungen durchgeführten FuE in hohem (und zunehmendem) Maße dem "faktischen Einfluß des Unternehmenssektors" unterliegen (so z.B. auch Ronge 1986 und Esser 1987), schätzen kritische Beobachter, daß auf "die Industrie- und die industrienahe Forschung ... etwa 80 Prozent der in der Bundesrepublik für FuE aufgewandten Finanzmittel entfallen [dürften]" (Simonis 1989: 47).

\section{Ambivalenz der Industrieforschung}

Forschungs- und Entwicklungseinrichtungen erbringen für die Unternehmen eine Vielzahl von Leistungen: Sie sollen Wissen für künftige Produkt- und Verfahrensinnovationen bereitstellen (Innovationsfunktion); sie haben die

3 Die Bildung von Durchschnitten erscheint jedoch sehr problematisch, wenn in den einschlägigen Studien die Personalangaben für einzelne Unternehmen zwischen $7 \%$ (Thyssen) und $46 \%$ (Boehringer/Ingelheim) schwanken (vgl. Hack 1990). 
Aufgabe, die wissenschaftlich-technologische Umwelt der Unternehmen zu beobachten, dort gefundene Entdeckungen und technologische Trends zu bewerten und ihre künftige Bedeutung zu prognostizieren (Monitoring-, Evaluations- und Prognosefunktion) sowie vor diesem Hintergrund strategische Unternehmensentscheidungen beratend zu unterstützen (Beratungsfunktion); von ihnen wird schließlich auch erwartet, daß sie Hilfestellungen bei kurzfristig auftretenden Problemen in der Konstruktion oder Produktion geben (Dienstleistungsfunktion) (vgl. Rosenberg 1990).

Mit all diesen Leistungen ist die Industrieforschung zunächst unzweifelhaft ein Teil des Wirtschaftssystems. Sie ist eine Unternehmensfunktion wie die Konstruktion, die Fertigung, der Vertrieb oder das Marketing. In dieser Funktion muß sie ökonomisch "verwertbare" Resultate liefern, müssen sich ihre Ergebnisse in "Zahlungen" (Luhmann) rechnen lassen. Darüber darf aber nicht vergessen werden, daß die Industrieforschung gleichzeitig auch Teil des Forschungssystems ist, genauso wie die Hochschulen oder die außeruniversitären staatlichen Forschungseinrichtungen. In dieser Funktion wird sie danach beurteilt, ob sie wissenschaftlich "wahrheitsfähige" Aussagen liefert. Charakteristisch für die Industrieforschung ist also, daß ihre wissenschaftlichen Leistungen in einem Organisationszusammenhang erbracht werden müssen, dessen Organisationszweck eindeutig nicht-wissenschaftlich definiert ist.

Die Konsequenzen dieser organisationsinternen Koexistenz unterschiedlicher systemischer Leistungsorientierungen und -erwartungen für die Industrieforschung blieben bislang umstritten. Unproblematisch erscheint diese Koexistenz in der Sicht der neueren Systemtheorie, die funktionale Teilsysteme lediglich über systemspezifische Kommunikationen definiert und nicht als "Seinsregionen" (Luhmann 1990: 86) begreift. Die Systemtheorie setzt darauf, daß Operationsketten "blitzschnell" umschalten können: "vom Rechtscode zum politischen Code, vom Wissenschaftscode zum Wirtschaftscode" (Luhmann 1990: 87). Andere Ansätze lösen die Koexistenz auf und behaupten die Dominanz einer der beiden Orientierungen. Am weitesten verbreitet sind dabei Instrumentalisierungsthesen. Diese schließen aus der organisatorischen Einbindung der Industrieforschung in die Unternehmen auf die "Vorherrschaft ökonomischer Regulative und Interessen"; industrielle Forschungs- und Entwicklungslaboratorien sind, nach diesem Verständnis, "ökonomisch definiert und kontrolliert" (Hack 1988: 94). Im Gegensatz hierzu geht die Theorie der wissenschaftlichen Selbstorganisation von der Autonomie von "Forschungsgruppen", ob an der Hochschule oder im Unternehmen, aus. Die Forschungsgruppe gilt als Gegenmodell zu hierarchischen Formen systemischer Integration, die "in der Art, wie sie sich selbstorganisiert, weitgehend freigestellt (sei); das 
gilt auch für hierarchisch durchorganisierte Laborbetriebe in der Industrie" (Krohn/ Küppers 1987: 26).

Allen diesen Ansätzen entgeht ein zentrales Merkmal der Industrieforschung: Die Ambivalenzen und Spannungen, die daraus resultieren, daß unterschiedliche Leistungserwartungen innerhalb einer Organisation erfüllt werden müssen. Auf solche Spannungen haben organisationssoziologische Studien (Kornhauser 1962, La Porte 1965, zuletzt Rammert 1988), die sich mit der Industrieforschung beschäftigten, seit langem und mit Nachdruck hingewiesen. Gerade in der Industrieforschung scheint das Grundproblem des Forschungsmanagements, die "Unvereinbarkeit gleichzeitiger Anforderungen" (Mayntz 1985: 31) in besonderer Schärfe aufzutreten, klaffen die Zielorientierungen von "Forschern" und "Managern" besonders weit auseinander. Der Abbau der daraus resultierenden interpersonellen Spannungen, die Schaffung "innovationsgerechter Arbeitsbedingungen", ist (noch immer) ein zentrales Thema des betriebswirtschaftlichen FuE-Managements (vgl. Bleicher 1990).

Allein schon die Persistenz dieses Spannungsverhältnisses weist darauf hin, daß wir es hier mit einem strukturellen Dilemma zu tun haben. In der Unternehmenspraxis bewegt sich die Industrieforschung beständig im Spannungsfeld zwischen "Instrumentalisierung" und "Autonomie". Denn ihre Funktion als Industrieforschung verlangt: "Forschungsleistungen müssen in die Zielsetzung des Unternehmens eingebunden und aus dieser abgeleitet werden" (v. Rensen 1989: 59). Zumindest als Norm wird postuliert: "Industrielle FuE muß nahtlos und rasch in die Fertigung einmünden; Spielraum für unabhängige schöpferische Aktivitäten kann [für das Unternehmen; EG/JH] gefährlich sein" (Danielmeyer 1990: 16). Aber gleichzeitig muß ihrer Funktion als Industrieforschung Rechnung getragen werden. Und das heißt, es "muß auch Freiraum geschaffen werden für neue Ideen, deren Umsetzung in eine marktfähige Leistung über das aktuelle Spektrum des Unternehmens hinausgeht" (v. Rensen 1989: 59). Dieses "Innovationsdilemma" (Rammert 1988) konkretisiert sich für die Unternehmen in zwei Formen: als Organisationsproblem und als Allokationsproblem.

Die Ambivalenz der Industrieforschung wirft für die Unternehmen zunächst Organisationsprobleme auf. Die Autonomie der FuE-Einrichtungen ist für sie zugleich notwendig und riskant. Sie ist notwendig, weil wissenschaftlichtechnologische Innovationen (auch im Unternehmen) häufig unintendiert und ungeplant entstehen (vgl. Rosenberg 1990: 169). Eine zu starke Instrumentalisierung der Industrieforschung gefährdet folglich ihre Leistungsfähigkeit. Mit der Gewährung von Freiräumen für die Forschung riskieren die Unternehmen freilich, daß sich ihre FuE-Abteilungen stärker an wissenschaftlichen "Oppor- 
tunitäten" orientieren und von den "lokalen Idiosynkrasien" (Knorr-Cetina 1984) ihrer Forschungskontexte leiten lassen als von den Interessen des Unternehmens. Nicht von ungefähr müssen die Unternehmen die "Bringschuld der Forschenden" immer einklagen, können sie bei ihnen eine "dem vermarktungsfähigen Ergebnis verpflichtete Arbeits- und Denkweise" (v. Rensen 1989: 61) nicht voraussetzen. Die "Gratwanderung zwischen der Freiheit des einzelnen und des notwendigen Controllings von Kosten und Terminen" (Kircher 1989) stellt die Unternehmen vor das Problem: Wie läßt sich die Organisation und Struktur der FuE-Einrichtungen im Unternehmen gestalten, so daß ihre Verselbständigung verhindert und ihre Leistungsfähigkeit erhalten bleibt?

Das strukturelle Dilemma der Industrieforschung, die Koexistenz unterschiedlicher Leistungsbezüge in den industriellen FuE-Einrichtungen, wirft für die Unternehmen außerdem Allokationsprobleme auf: Sollen Forschungsleistungen überhaupt intern erbracht werden? Wieviel soll in die interne FuE investiert werden? Wie kann die Verwendung der investierten Mittel effektiv kontrolliert werden? Keine dieser Fragen ist für die Unternehmen eindeutig zu beantworten. Dafür gibt es mehrere Gründe.

Zunächst darf nicht vergessen werden, daß sich die Unternehmen die Leistungen ihrer FuE-Einrichtungen nicht immer vollständig aneignen können (Arrow 1962). Das intern generierte Wissen kann nicht nur vom Wissenschaftssystem genutzt werden, sondern auch von anderen Unternehmen. Durch Geheimhaltungsvorschriften, Publikationsrestriktionen oder das Patentrecht können diese externen Effekte von unternehmensinterner FuE reduziert werden, gänzlich ausgeschlossen werden können sie aber nicht.

Hinzu kommt, daß die Kosten der Industrieforschung nicht exakt kalkuliert werden können, weil deren Leistungen sachlich und zeitlich diffus sind. Je weiter sich die Industrieforschung von konkreten Anwendungsbezügen entfernt, desto schwieriger wird es, sie sachlich auf die am Markt orientierten Unternehmensbereiche zu beziehen. Es bleibt immer ein "Restrisiko", ob Forschungsleistungen zu marktfähigen und marktgängigen Produkten führen. Zudem unterscheidet sich der Zeithorizont der Industrieforschung (in der Regel fünf bis zehn Jahre) deutlich von dem anderer Unternehmensbereiche. Damit muß vielfach unbestimmt bleiben, wann sich Forschungsleistungen rechnen werden. Industrieforschung muß sich aus diesem Grund häufig mit Leistungsversprechungen legitimieren, deren tatsächlicher Nutzen kurzfristig kaum zu ermitteln ist.

Schließlich stehen die Unternehmen vor dem Problem, daß nicht alle Kosten der Industrieforschung betriebswirtschaftlich gerechnet werden dürfen. FuE- 
Abteilungen müssen nicht nur den von Unternehmen intern generierten Bedarf abdecken, sie müssen auch ungewisse (wissenschaftlich-technologische) Entwicklungen in der Umwelt der Unternehmen wahrnehmen und verarbeiten können. Diese Resonanz- und Absorptionsfähigkeit (Cohen/ Levinthal 1990) setzt redundante Kapazitäten voraus, die in kurzfristigen Kostenrechnungen als "unnötige" Kosten behandelt werden. Auch an diesem Punkt befinden sich die Unternehmen in einem strukturellen Dilemma. Wenn sie ihren egoistischen, kurzfristigen Nutzenkalkülen folgen, dann laufen sie Gefahr, zu wenig in die interne FuE zu investieren. Suspensieren sie solche Kalküle jedoch zugunsten langfristiger Nutzenerwartungen, dann riskieren sie, zu viel in die interne FuE zu investieren.

Ob es sich um Organisationsprobleme handelt oder um Allokationsprobleme, das industrielle FuE-Mangement findet sich ständig in Entscheidungssituationen, in denen jede Lösung ihrerseits wiederum spannungs- und problemgeladen ist. Mehr noch als in staatlichen Forschungseinrichtungen gilt hier, "daß es offenbar keine in sich unproblematischen Organisationslösungen gibt" (Mayntz 1985: 30). Dieses strukturelle Dilemma des industriellen FuE-Managements hat steuerungstheoretisch eine doppelte Konsequenz: Es findet als interne Steuerungsnotwendigkeit in den Unternehmen seinen Niederschlag; und es eröffnet gleichzeitig externe Steuerungschancen für den Staat.

\section{Steuerungsprobleme des industriellen Forschungsmanagements}

Jede in steuerungstheoretischer Perspektive durchgeführte konkrete Analyse industrieller FuE-Aktivitäten hat zunächst davon auszugehen, daß diese in jeweils spezifischer Weise in den Organisationszusammenhang von Unternehmen eingebunden sind und sich nicht von ihren horizontalen und vertikalen Bezügen zu anderen Unternehmenseinheiten isolieren lassen. Die Bandbreite möglicher und existierender Formen der betrieblichen Integration kann zudem nicht hinreichend von zugespitzten Vorstellungen der vollständigen "Funktionalisierung", bzw. "Instrumentalisierung" der FuE-Aktivitäten oder ihrer vollständigen "Selbständigkeit", bzw. "Autonomie" wiedergegeben werden (vgl. auch Zündorf/ Grunt 1982 und Rammert 1988). Präzisere Analysen sind steuerungstheoretisch notwendig, weil spezifische Unternehmensstrukturen und -strategien in entscheidendem Maße die Ausprägungen der Strukturmerkmale unternehmerischer FuE-Aktivitäten - insbesondere den Grad ihrer Autonomie im Unternehmensverbund - und damit die Möglichkeiten und Bedingungen ihrer externen politischen Steuerbarkeit beeinflussen. 
Abzugehen ist grundsätzlich von der Vorstellung, daß es sich bei Unternehmen um unitarische und hierarchisch koordinierte korporative Akteure handelt. Stattdessen müssen Unternehmen als komplexe Organisationen begriffen werden (vgl. March 1988), in denen auch FuE-bezogene Entscheidungen auf sehr unterschiedliche Arten getroffen werden. Als "hybride Organisationen" (Powell 1987, Bradach/ Eccles 1989) nutzen Unternehmen auch im FuE-Bereich eine Mehrzahl von Koordinierungs-, Kontroll- und Regulierungsmechanismen.

Sicherlich existieren Bereiche und Probleme, für deren Bearbeitung oder Lösung hierarchische Entscheidungsmodi dominieren, Entscheidungen von den Unternehmens"spitzen" getroffen werden und FuE-Abteilungen tendenziell nicht am Entscheidungsprozeß beteiligt sind; dies dürfte beispielsweise gelten, wenn Unternehmen die Aufgabe ganzer Unternehmensbereiche - und damit FuE-Schwerpunkte - auf der Basis "forschungsfremder" Kriterien beschließen (so geschehen im aktuellen Sanierungsprozeß bei Philips). In aller Regel praktizieren Unternehmen allerdings Verfahren der - entsprechend etwa dem jeweiligen finanziellen Umfang einzelner FuE-Projekte - abgestuften hierarchischen Steuerung der FuE-Aktivitäten, an dessen einem Ende Vorstandsbeschlüsse zwingend sind, an dessen anderem Ende allerdings autonome Entscheidungen der FuE-Abteilungen stehen.

Daneben werden Aktivitäten industrieller FuE-Abteilungen über weite Strekken auch durch Marktmechanismen gesteuert. So wird ein Großteil der Entwicklungsarbeiten in Unternehmen des Maschinenbaus im Rahmen von Kundenaufträgen abgewickelt und dadurch eben extern durch die jeweiligen spezifischen Kundenwünsche gesteuert. ${ }^{4}$ Vielfach nähern sich unternehmensinterne Beziehungen, etwa zwischen Zentrallabors und bereichsbezogenen Entwicklungslabors, Marktbeziehungen dadurch an, daß Zentrallabors mit der einschlägigen wissenschaftlich-technischen Umwelt des Unternehmens um die Aufträge der Entwicklungsabteilungen konkurrieren müssen.

Nicht zuletzt fallen zahlreiche FuE-bezogene Entscheidungen in unternehmensinternen Verhandlungsgremien. So obliegt die bereichsübergreifende Koordination der FuE-Aktivitäten in zahlreichen Großunternehmen Ausschüssen, in denen die jeweils für FuE zuständigen Repräsentanten der Unternehmensbereiche - oft gleichberechtigt - mit dem Leiter des zentralen FuE-Bereichs vertreten sind (so etwa bei der Siemens AG; vgl. auch de Pay 1989). Um den innerbetrieblichen Technologietransfer zu gewährleisten, werden an

4 Vgl. etwa Zörgiebel 1983. Zu den strukturellen und aktuellen Grenzen dieses Modells der FuE-Steuerung vgl. Häusler 1990. 
vielen Nahtstellen des unternehmensinternen Innovationssystems - so am Übergang von Entwicklungs- zu Produktionsabteilungen - konsensorientierte Verhandlungslösungen, beispielsweise in Projektgruppen als "mediatisierenden Gremien" (Rammert 1988: 188), angestrebt.

Neben der Existenz unterschiedlicher Entscheidungsstrukturen und -prozeduren in einzelnen Unternehmen (oder Unternehmen in spezifischen Größenklassen, Branchen etc.) verweisen auch unterschiedliche Finanzierungsmodi auf die Notwendigkeit, den jeweiligen Grad an Autonomie einer spezifischen industriellen FuE-Einrichtung hinreichend differenziert zu ermitteln. Eher gering dürfte dieser sein, wenn Entwicklungsarbeiten zu einem wesentlichen Teil im Wege der Auftragsentwicklung durch den Kunden finanziert werden, da hier die Auftragsakquisition vornehmlich über den Vertrieb erfolgt. Diese Situation kann sich im Sinne eines erweiterten Spielraums für die FuE-Abteilung etwa dann verändern, wenn diese in der Lage ist, bzw. von der Unternehmensleitung dazu aufgefordert wird, extern finanzierte Auftragsforschung einzuwerben (wie im Falle des Porsche-Entwicklungszentrums geschehen).

In Großkonzernen existieren in der Regel sehr differenzierte Modi der Finanzierung der internen FuE-Aktivitäten. In "multiple funding systems" (Bleicher 1990: 167) werden unterschiedliche FuE-Aufgaben in unterschiedlicher Weise, also etwa aus verschiedenen Quellen und unter Maßgabe unterschiedlicher Kriterien, finanziert. So werden beispielsweise die Forschungstätigkeiten eines Elektrounternehmens zu 50\% eigenfinanziert, wobei diese Mittel vom Gesamtunternehmen kommen oder aus umsatzabhängigen Abgaben der Produktbereiche resultieren; weitere $25 \%$ erbringt die Unterstützung durch die öffentliche Hand, während die restlichen $25 \%$ schließlich aus Entwicklungsaufträgen der einzelnen Entwicklungsabteilungen der Produktbereiche resultieren. Die Finanzierung des zentralen Forschungslabors eines anderen Elektrokonzerns erfolgt, wohl nicht zuletzt in Reaktion auf das oben beschriebene strukturelle Innovationsdilemma, auf zwei Arten. Eine zentrale Umlage $(0,5-1 \%$ des Umsatzes) finanziert einen Bereich, in dem das Zentrallabor in Eigenverantwortung die Prioritäten setzt. Daneben vergeben die Unternehmensbereiche Aufträge, die sie bis zu $100 \%$ finanzieren. Modellhaft verteilen sich diese Finanzierungsmodi wie folgt: Vorfelduntersuchungen und Exploration (25\% der Aktivitäten des Zentrallabors) werden zu 100\% über die Umlage finanziert; Feasibility-Untersuchungen (50\%) werden jeweils zur Hälfte vom Zentralbereich und von den Unternehmensbereichen finanziert; TransferProjekte (25\%) werden schließlich zu 100\% von den Bereichen getragen. Derart elaborierte Finanzierungssysteme versuchen einerseits sicherzustellen, daß marktnahe Entwicklungsaufgaben in enger Abstimmung mit den Produktions- und Vertriebsabteilungen der jeweiligen Unternehmensbereiche erfolgen; sie insti- 
tutionalisieren gleichwohl auch einen gewissen Freiraum etwa für grundlagenorientierte Forschungsarbeiten.

Einen ähnlichen Effekt der differenzierenden Problembearbeitung können unterschiedliche Formen der Organisation der unternehmensinternen FuEAktivitäten zeitigen. Wenn es um die organisatorische Gestaltung des industriellen FuE-Prozesses geht, steht in der Regel die Frage nach der Aufteilung in zentrale und dezentrale Einheiten im Vordergrund; dabei existieren insbesondere in Großunternehmen - zumeist beide Organisationsformen. Eine starke organisatorische Konzentration von FuE-Aktivitäten findet sich lediglich in streng funktional gegliederten Unternehmen - vorausgesetzt, daß eine eigenständige FuE-Abteilung existiert; in Unternehmen ohne eigenständige FuE-Abteilung, etwa in manchen mittelständischen Unternehmen des Maschinenbaus, sind FuE-Aktivitäten - dann vorwiegend Entwicklungstätigkeiten dezentral in die Konstruktionsabteilungen integriert. In nach Produktgruppen divisionalisierten Großkonzernen existieren komplexe FuE-Organisationsstrukturen mit unternehmensweit zentralisierten grundlagenorientierten Forschungslabors, zentralen Labors der Grundlagenentwicklung in den einzelnen Unternehmensbereichen und schließlich sehr weitgehend dezentralisierten Entwicklungslabors.

Die geraffte Darstellung der auftretenden Variationen in den Entscheidungsstrukturen, Finanzierungsmodi und Organisationsformen unternehmensinterner FuE-Aktivitäten läßt zusammenfassend den Kern der Strategie erkennen, mit der Unternehmen das im vorigen Abschnitt herausgearbeitete Innovationsdilemma zu lösen trachten: Problemdifferenzierung und Differenzierung der unternehmensinternen Forschungssysteme. Als Resultat des Bestrebens, das strukturelle Steuerungsproblem des industriellen FuE-Managements kleinzuarbeiten und im Anschluß differenziert zu bearbeiten, entstehen komplexe FuE-Strukturen in den Unternehmen.

Modellhaft lassen sich die Elemente dieser Struktur auf einem Kontinuum abbilden. Am einen Ende wird grundlagenorientierte Forschung, per unternehmensweiter Umlage sozusagen grundfinanziert, in einem weitgehend autonomen Zentrallabor durchgeführt (die fünf Forschungszentren der IBM, u.a. jenes in Rüschlikon, gleichen diesem Typus). Am anderen Ende des Kontinuums führen dezentrale Entwicklungslabors Aufträge von Konstruktions-, Produktions- und Vertriebsabteilungen aus, die von diesen finanziert und inhaltlich weitgehend determiniert werden.

Die Komplexität und Variabilität realer Binnenstrukturen industrieller FuE sind von steuerungstheoretischem Interesse, da sie - wie im nächsten Kapitel 
zu zeigen sein wird - Konsequenzen für die jeweilige externe Beeinflußbarkeit industrieller FuE-Aktivitäten haben.

Neben der Variation von FuE-Binnenstrukturen zwischen Unternehmen (oder Unternehmen unterschiedlicher Branchenzugehörigkeit oder Größe) treten deutliche inter-temporale Variationen der FuE-Binnenstrukturen auf, da Unternehmen nahezu ständig mit Verfahrens- und Organisationsreformen versuchen, das strukturelle Innovationsdilemma zu bewältigen. ${ }^{5}$ Erklärbar sind diese ständigen Organisationsreformen und -debatten mit der Einsicht, daß den Unternehmen auf Dauer gestellte eindeutige Entscheidungen strukturell unmöglich sind, weil jede "Organisationsform, jedes Finanzierungsverfahren [etc.] an irgendeiner Stelle auf einem spannungsgeladenen Kontinuum zu liegen (scheint), dessen Pole zwei gegensätzliche Werte maximieren. Dies ist der Grund, warum es keine unproblematischen Lösungen gibt, sondern nur den Versuch, den Tiger zu reiten, die Spannungen auszugleichen und das Abgleiten in das eine oder andere Extrem zu verhindern" (Mayntz 1985: 31). Bestimmbare optimale Lösungen existieren für das industrielle FuE-Management z.B. nicht, wenn es um die Wahl zwischen dem Aufbau hinreichender redundanter FuE-Kapazitäten und der Senkung der FuE-Aufwendungen, dem Aufbau interner FuE-Kapazitäten (und damit weitreichender technologischer Autonomie) und der (eventuell kostensenkenden) Nutzung externer Kapazitäten, oder zwischen kurzfristiger Marktorientierung und langfristiger Orientierung am technologischen Potential des Unternehmens geht.

Auch auf der (Meta-) Entscheidungsebene des industriellen FuE-Managements, wo es um die Festlegung der FuE-Strukturen geht (also etwa um die Verteilungsrelation zwischen den oben beschriebenen Typen unternehmensinterner Forschungseinrichtungen), existieren unterschiedliche Entscheidungsstrukturen und -prozesse. Hierarchische Festlegungen der Forschungsstrategie und der FuE-Aufbauorganisation dürften eher selten anzutreffen sein; vorstellbar sind sie dennoch etwa in Kleinunternehmen oder in Unternehmen mit starken Gründerpersönlichkeiten. Nixdorf wäre ein Fall, der zum einen wahrscheinliche Konsequenzen patriarchaler Unternehmensführung für die (begrenzte) technologische Adapationsfähigkeit eines Unternehmens illustriert und zum anderen auch erwartbare Reaktionen der FuE-Abteilungen deutlich macht: gegen den erklärten Willen des Unternehmensleiters, ausschließlich kundeninduzierte Entwicklungsarbeiten durchzuführen, engagierte sich die FuE-Abteilung "subversiv" in grundlagenorientierten Forschungsprojekten weitgehend jener des Forschungsmanagements in wissenschaftlichen Einrichtungen (vgl. Mayntz 1985: 7). 
zum Teil in Allianzen mit externen Forschungseinrichtungen und Förderstellen und unter Einsatz staatlicher Fördergelder. Auch in anderen Unternehmen ist die Existenz solcher sogenannter "U-Boot-Projekte" - trotz oder besser: wegen der zunehmenden Versuche, auch Forschungslabors strenger hierarchisch zu steuern - hinreichend bekannt.

In der Regel sind auch FuE-bezogene Strukturentscheidungen wohl eher Ergebnis "mikropolitischer" Auseinandersetzungen und Verhandlungen im Unternehmen (vgl. Küpper/ Ortmann 1986; 1988), das in dem Sinne ein "politisches System" darstellt, "daß jede Funktionsgruppe auch eine Interessengruppe ist, die versucht in strategischem Gegen- und Miteinander, ihre partikularen Interessen durchzusetzen" (Zündorf/ Grunt 1982: 22; vgl. auch March 1988). An forschungsstrategischen und -organisatorischen Entscheidungen als Ergebnis unternehmensinterner Konflikte, Verhandlungen und Kompromisse sind längst "nicht nur die jeweiligen speziellen FuE-Bereiche beteiligt, sondern darüber hinaus u.a. auch die Marketing- und Verkaufsabteilungen, die die Anforderungen des Marktes in den Innovationsprozeß einbringen, die Controlling- und Wertanalyseabteilungen, die die Wirtschaftlichkeit der Forschungs- und Entwicklungstätigkeiten überwachen, die Produktions- und Fertigungsabteilungen, die die entwickelten Produkte schließlich mit dem gegebenen bzw. anzupassenden Produktionsapparat herstellen müssen, die Organisations- und Planungsabteilungen, die dafür sorgen, daß die komplizierten und prekären Arbeits-, Abstimmungs- und Entscheidungsprozesse nicht das Organisationsgefüge sprengen, sondern zielstrebig und koordiniert ablaufen" (Zündorf/ Grunt 1982: 12).

\section{Chancen und Grenzen staatlicher Techniksteuerung in der Industriefor- schung}

Die Ambivalenz industrieller FuE und die Probleme ihrer unternehmensinternen Organisation machen externe Einflußnahmen zugleich möglich und schwierig. Die externe Steuerung von Industrieforschung wird möglich, weil Unternehmen ihre FuE-Einrichtungen nicht einfach "instrumentalisieren" und eindeutig und durchgängig in den Produktionszusammenhang integrieren können. Die der Industrieforschung inhärenten Ambivalenzen konstituieren Freiheitsgrade für Entscheidungen der Unternehmen bei der internen Organisation von FuE; und diese Organisationsentscheidungen stecken den Handlungsspielraum für die FuE-Einrichtungen ab. In diesem Zusammenhang kann staatliche Steuerung positive oder negative Anreize zur internen Auswahl von Optionen geben. Wohlgemerkt, bei dieser Art der staatlichen Steuerung geht es nicht darum, abstrakte Verwertungslogiken der Ökonomie außer Kraft zu 
setzen, sondern konkrete Entscheidungsspielräume und -dilemmata der Unternehmen für selektive Interventionen zu nutzen.

Gleichzeitig erschweren die komplizierten Binnenstrukturen der Unternehmen eine gezielte externe Einflußnahme. Staatliche Versuche, FuE-bezogene Unternehmensentscheidungen direkt zu beeinflussen, müssen nicht nur die Komplexität und Variabilität von FuE-Strukturen in Unternehmen und deren jeweilige Konsequenzen im Hinblick auf die Autonomie der FuE-Einrichtungen berücksichtigen; sie müssen auch die komplexen Konflikt- und Verhandlungsprozesse zur Kenntnis nehmen, als deren Resultat sich das unternehmensinterne Forschungssystem etabliert und immer wieder restrukturiert. Die Resonanz auf staatliche Politiken ist dann nicht nur abhängig von den strategischen Dispositionen des Unternehmens, sondern auch von den strategischen Freiheitsgraden von Forschungseinrichtungen und Forschergruppen in Unternehmen, deren spezifischem Förderbedarf sowie den unternehmensinternen Verhandlungspositionen und Machtkonstellationen.

Wenn wir die externe Steuerbarkeit industrieller FuE zunächst anhand der internen Autonomie der FuE-Einrichtungen einerseits, ihres Förderbedarfs andererseits bemessen, dann können wir zugespitzt vier Grundkonstellationen unterscheiden (vgl. Schaubild 2):

Schaubild 2: Typologie staatlicher Steuerbarkeit unternehmensinterner FuE-Aktivitäten

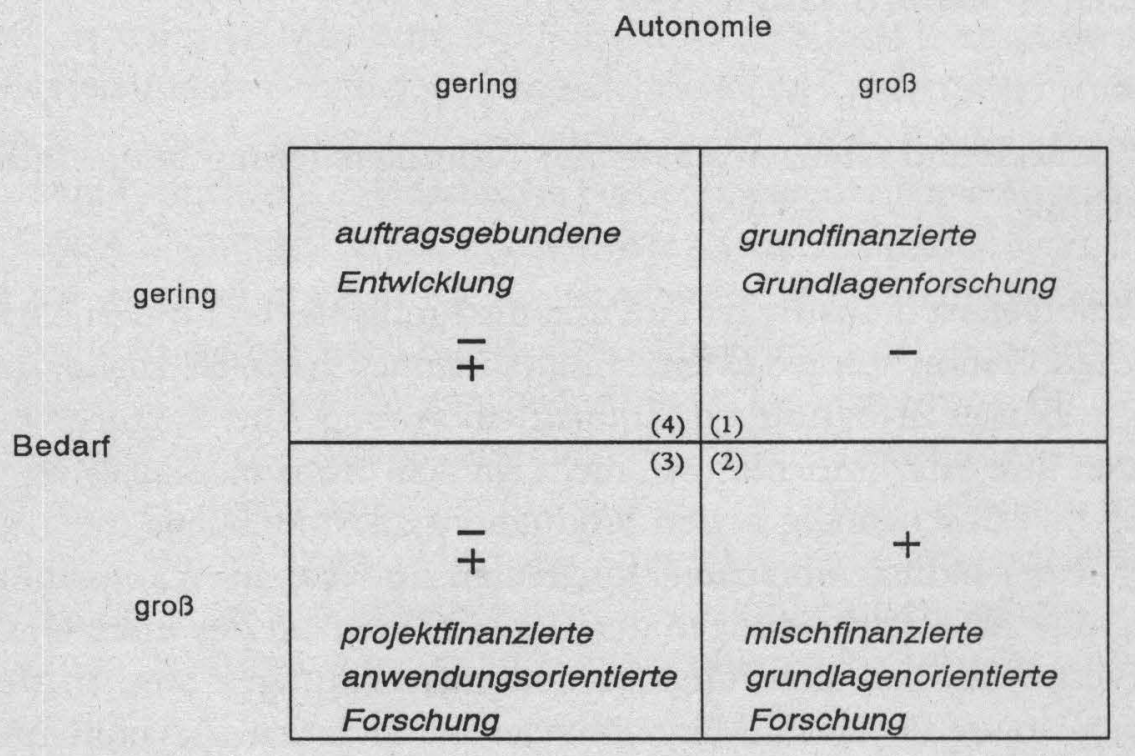


(1) Geringe staatliche Steuerungschancen bestehen bei Forschungseinrichtungen mit großer Autonomie und geringem Förderbedarf (Feld 1). Diese sind typischerweise in der grundfinanzierten Grundlagenforschung zu finden. Die Steuerungsbarriere besteht in diesem Fall nicht in der Unselbständigkeit der Forschung in den Unternehmen, sondern in ihrer großen Selbständigkeit. Zwar spielen die (langfristigen) strategischen Kalküle der Unternehmen bei der Errichtung (und Schließung) einer solchen Forschungseinrichtung die entscheidende Rolle; bei der Ausgestaltung und Durchführung ihres Forschungsprogramms ist diese aber weitgehend unabhängig. Da diese Forschungseinrichtungen ihren Ressourcenbedarf zum Teil vollständig projektungebunden intern decken, lassen sie sich mittels finanzieller staatlicher Anreize kaum steuern. Ihre primären externen Bezugspunkte besitzen solche Forschungseinrichtungen in der wissenschaftlichen Umwelt des Unternehmens. Der Staat kann den Unternehmen in diesem Fall zum Beispiel Anreize bieten zum Aufbau, zum Erhalt oder zur Erweiterung von Forschungseinrichtungen, um so Unterinvestitionen von Unternehmen in die Grundlagenforschung gegenzusteuern; oder er kann versuchen, den Wissenstransfer zwischen staatlichen und unternehmerischen Forschungseinrichtungen $\mathrm{zu}$ verbessern.

(2) Die günstigsten staatlichen Steuerungschancen bestehen dort, wo industrielle Forschungseinrichtungen zwar über eine hinreichend große Autonomie verfügen, um zumindest einen Teil ihrer Forschungsthemen und -projekte selbst wählen zu können, ihr Ressourcenbedarf aber nicht ausschließlich durch eine interne Grundfinanzierung gedeckt ist (Feld 2). Diesen Fall finden wir in mischfinanzierten Einrichtungen der angewandten Grundlagenforschung. In diesen (zumeist zentral organisierten) Forschungseinrichtungen sind zwar "Kerntechnolgien" und "Schlüsselprojekte" durch das Unternehmen und dessen (längerfristige) Technologiestrategie vorgegeben und intern finanziert; daneben haben diese Forschungseinrichtungen aber auch die Möglichkeit, in begrenztem Umfang Projektmittel zu akquirieren, zum Beispiel um Forschungsfelder $\mathrm{zu}$ komplettieren, alternative Forschungspfade $\mathrm{zu}$ sondieren u.a. Und in diesem Zusammenhang spielen nicht nur interne Projektaufträge durch Unternehmensbereiche eine Rolle, sondern auch externe Forschungsaufträge und staatliche Fördermittel.

(3) In Fällen, in denen FuE-Einrichtungen zwar Förderbedarf aufweisen, aber nicht die erforderliche Autonomie besitzen, um Forschungsthemen und -projekte selbständig zu bestimmen, ist eine staatliche Steuerung zwar nicht grundsätzlich ausgeschlossen, aber abhängig von zusätzlichen internen Bedingungen (Feld 3). Diese Konstellation trifft in der Regel bei (dezentral organisierten) anwendungsorientierten Forschungseinrichtungen zu, deren Tätigkeit durch die Unternehmensstrategie vorgegeben ist und die sich überwiegend 
aus projektgebundenen Zuweisungen der Produktbereiche des Unternehmens finanzieren. Die interne Resonanz auf Förderangebote des Staates hängt dann entscheidend $\mathrm{ab}$ von den strategischen Dispositionen des Unternehmens bzw. seiner Produktbereiche. Staatliche Fördermöglichkeiten werden häufig dann wahrgenommen, wenn technologische "Umbrüche" kurzfristig die internen Kapazitäten der Unternehmen überfordern, die Forschungsleistungen aus strategischen Gründen aber notwendig erscheinen. Die Integration der Elektronik im Maschinenbau, der Einstieg in die optische Nachrichtentechnik in der Telekommunikation oder der Übergang zu integrierten Schaltkreisen in der Mikroelektronik wären Beispiele hierfür. Die Notwendigkeit, an die unternehmensstrategischen Präferenzen anschlußfähig zu sein, bringt in diesem Fall für die staatliche Förderpolitik jedoch die Gefahr mit sich, lediglich auf Vorgaben der Unternehmen zu reagieren und so ihre selektive Steuerungswirkung zu verlieren.

(4) Auch der Fall, in dem die FuE-Einrichtungen in Unternehmen weder über die Autonomie verfügen, um auf staatliche Anreize eingehen zu können, noch einen Bedarf nach externer Förderung aufweisen, ist differenziert zu betrachten (Feld 4). Typisch hierfür sind Entwicklungsabteilungen, deren Aktivitäten weitgehend durch Kunden oder nachgelagerte Produktbereiche im Unternehmen bestimmt und finanziert werden. Durch die enge Kopplung zwischen Kunden, Vertrieb, Produktion und Entwicklung haben externe Interventionen, z.B. mittels öffentlicher Förderangebote, nur geringe Chancen. Diese Situation war lange Zeit charakteristisch für den bundesdeutschen Maschinenbau. Erst als diese Branche unter Innovationsdruck kam und sich die Kundenorientierung der Entwicklungsabteilungen lockerte, ergaben sich dort Ansatzpunkte für die staatliche Förderpolitik (vgl. Häusler 1990; 1991). Groß sind die staatlichen Steuerungsmöglichkeiten dagegen dann, wenn der Staat in dieser Konstellation selbst als bedeutender Kunde auftritt, sei es durch die Vergabe von Entwicklungsaufträgen an die Unternehmen oder als Abnehmer ihrer Produkte. Die Kommunikationstechnik wäre ein Beispiel dafür, wie der Staat als wichtigster (und oftmals einziger) Kunde der Unternehmen über seine Beschaffungspolitik Einfluß auf deren Entwicklungsaktivitäten nehmen kann.

Diese Typologie macht deutlich, in welchem Maße die staatliche Steuerbarkeit von Industrieforschung kontingent ist. Da wir es vor allem in den Großunternehmen in der Regel mit komplexen, ausdifferenzierten Forschungssystemen zu tun haben, dürfte die externe Steuerbarkeit industrieller FuE nicht nur innerhalb der Unternehmen zwischen den verschiedenen FuE-Einrichtungen, sondern auch zwischen den Unternehmen und Branchen beträchtlich variieren. 
Schließlich ist zu berücksichtigen, daß auch in den günstigsten Fällen externer Steuerung diese immer nur vermittelt über die internen Machtpositionen und Verhandlungsprozesse erfolgen kann. Staatliche Steuerung - wie auch ihre politikwissenschaftliche Analyse - muß in Rechnung stellen, daß die betriebliche FuE-Organisation ein spannungsgeladenes, umkämpftes Terrain konstituiert. Vor allem im zweiten, aber auch im dritten hier skizzierten Typus konfligieren Autonomiebestrebungen von Forschergruppen und Forschungseinrichtungen häufig mit Instrumentalisierungsversuchen durch die Unternehmensbereiche und -leitungen. Mit seinen Förderprogrammen wird der Staat in diesen Fällen zum Akteur in der "Mikropolitik" des Unternehmens, öffentliche Fördermittel können (und müssen) die Machtspiele und -positionen in unternehmensinternen Verteilungskonflikten verändern.

Die Aussicht auf externe Ressourcen kann die internen Durchsetzungschancen von FuE-Projekten entscheidend verbessern. Vermittelt über die unternehmensinternen Machtspiele erhalten öffentliche Förderinstanzen dadurch zumindest marginale Möglichkeiten zur Beeinflussung der Inhalte von FuEArbeiten in Unternehmen. Die Wirkung dieser selektiven Intervention kann verstärkt werden, indem die Vergabe von Fördermitteln an zusätzliche inhaltliche und prozedurale Vorgaben geknüpft wird. In der Praxis ist dabei nicht auszuschließen, daß ein FuE-Projekt auch ohne öffentliche Fördermittel durchgeführt würde, und je größer das Interesse öffentlicher Förderinstanzen an industriell unmittelbar verwertbaren Forschungsergebnissen ist, desto wahrscheinlicher wird dieser Fall sein. Solche "Mitnahme-Effekte" sind jedoch nicht zwangsläufig. Staatliche Förderprogramme können nicht nur mit dem Opportunismus der Unternehmen rechnen, sondern auch mit dem Opportunismus der Forscher in Unternehmen. Auch die Präferenzen von Forschergruppen in Unternehmen sind "gelegenheitserzeugt" (Knorr-Cetina 1984: 80); öffentliche Förderprogramme müssen dann lediglich anschlußfähig sein an die lokalen Forschungskontexte und nicht vollständig mit vorgegebenen Präferenzen harmonieren.

Da die Verfügung über Ressourcen ein zentrales Kriterium der Autonomie von FuE-Einrichtungen darstellt, ist schließlich evident, daß staatliche Fördermittel - ungeachtet der damit verbundenen inhaltlichen Vorgaben - auch die Position ihrer Empfänger im Unternehmen beeinflussen. Grundsätzlich wird dadurch die "Bringschuld" der Industrieforschung gegenüber dem Unternehmen gemindert. Wichtiger aber noch dürften die nicht-monetären Implikationen sein. Mit der Annahme staatlicher Fördermittel werden unternehmerische Forschungsleistungen an externe Erwartungen und Auflagen gebunden; und diese externen Verpflichtungen begrenzen die internen Möglichkeiten des FuE-Managements bzw. der Unternehmensleitungen, auf den Inhalt und den 
Verlauf von FuE-Projekten Einfluß zu nehmen. Ein öffentlich gefördertes Projekt kann z.B. nicht so leicht gestoppt oder gekürzt werden wie ein ausschließlich intern finanziertes; zusätzliche Leistungserwartungen oder interne Fristen können mit Verweis auf externe Vorgaben zurückgewiesen werden. Solche Effekte werden noch verstärkt, wenn die öffentliche Förderung gebunden ist an die Kooperation mit wissenschaftlichen Einrichtungen oder anderen Unternehmen. Auch in diesem Fall können Verpflichtungen gegenüber Dritten die Autonomie eines FuE-Projekts innerhalb des Unternehmens stärken.

Staatliche Förderung kann die Autonomie der Akteure jedoch auch in die entgegengesetzte Richtung beeinflussen. Sie muß die FuE-Abteilungen nicht notwendig von kurzfristigen Ergebnisverpflichtungen gegenüber den Unternehmen entlasten, sie kann sie damit auch noch zusätzlich belasten. Das ist insbesondere in solchen Förderprojekten der Fall, deren Ziel primär die kurzfristige Stärkung der industriellen Wettbewerbsposition der Unternehmen ist. Hier ist die Autonomie der FuE-Einrichtungen nicht nur ein Problem für die Unternehmen, sondern auch für die staatlichen Förderinstanzen. Wenn FuEProjekte nicht unmittelbar in die Produktstrategie der Unternehmen eingebunden sind, dann riskiert auch der Staat, Projekte ohne unmittelbaren industriellen Nutzen $\mathrm{zu}$ fördern und so sein Steuerungsziel zu verfehlen.

\section{Forschungsperspektiven}

Welche Konsequenzen ergeben sich aus dieser Analyse für die weitere politikwissenschaftliche Forschung zur staatlichen Techniksteuerung? Zunächst dürfte deutlich geworden sein, daß die externe Steuerbarkeit der Industrieforschung weder umstandslos vorausgesetzt, noch einfach bestritten werden kann. Die Komplexität und Variabilität der unternehmensinternen FuE-Strukturen eröffnet staatlichen Akteuren durchaus die Möglichkeit der gezielten Intervention und der selektiven Partizipation. Aufgrund der Vielzahl kontingenter Bedingungen muß die Steuerbarkeit der Industrieforschung aber immer von Fall zu Fall empirisch ermittelt werden. Notwendig sind daher zum einen Fallstudien zu den Makrokonstellationen staatlicher Steuerungspotentiale, und zum anderen Untersuchungen, die sich mit den Mikroprozessen und -politiken staatlicher Intervention beschäftigen. $\mathrm{Zu}$ beidem besteht noch erheblicher Forschungsbedarf.

Forschungsbedarf besteht außerdem zu der Frage, ob die staatlichen Akteure überhaupt in der Lage sind, die Steuerungschancen, die ihnen das industrielle FuE-System bietet, effektiv zu nutzen. Angesichts der Komplexität und Variabilität der Forschungsorganisation in Unternehmen benötigt erfolgreiche staat- 
liche Techniksteuerung nicht nur Kenntnisse über technologische Trends und den Förderbedarf von Branchen, sondern auch detaillierte Informationen über die "internen Zustände" von Unternehmen. Je präziser die Kenntnisse der FuE-Systeme in Unternehmen und der unternehmensinternen Verhandlungspositionen und Machtkonstellationen sind, desto größer ist die Chance, auch mit geringen Mitteln Einfluß auf die internen Selektionsprozesse zu gewinnen. Fehlen solche Informationen, dann laufen auch voluminöse Förderprogramme Gefahr, ihre intendierte Wirkung zu verfehlen. Die Höhe staatlicher FuE-Zuwendungen an die Industrie muß also nicht immer ein zuverlässiger Indikator für die "Stärke" des Staates in der Forschungs- und Technologiepolitik sein. Umso wichtiger ist es deshalb, systematischer zu untersuchen, wie die staatlichen Akteure ihr Steuerungswissen generieren (können). In diesem Zusammenhang müßten insbesondere die formellen und informellen Politiknetzwerke zwischen Staat und Industrie in der Forschungs- und Technologiepolitik detaillierter erforscht werden.

Schließlich ist die Relevanz erfolgreicher staatlicher Interventionen in die Industrieforschung präziser zu bestimmen. Gerade weil die staatliche Politik im besten Fall nur punktuell und selektiv in das FuE-System der Unternehmen eingreifen kann, stellt sich die Frage, ob diese Mikrointerventionen signifikante Makroeffekte zur Folge haben können. Wäre dies nicht der Fall, dann müßte staatliche Techniksteuerung auch unter günstigsten Bedingungen marginal bleiben. 


\section{Literatur}

Alemann, Ulrich von et al. 1988: Technologiepolitik: Grundlagen und Perspektiven in der Bundesrepublik Deutschland und Frankreich. Frankfurt a. M.: Campus.

Arrow, Kenneth J., 1962: Economic Welfare and the Allocation of Resources for Invention. In: Richard R. Nelson (Hrsg.), The Rate and Direction of Inventive Activities: Economic and Social Factors. Princeton: Princeton University Press, 609-626.

Biegel, Udo Rudolf, 1987: Kooperation zwischen Anwender und Hersteller im Forschungs- und Entwicklungsbereich. Frankfurt a. M.: Lang.

Bleicher, Frank, 1990: Effiziente Forschung und Entwicklung. Wiesbaden: Deutscher Universitätsverlag.

BMFT, 1990: Faktenbericht 1990 zum Bundesbericht Forschung 1988. Bonn: BMFT.

Bradach, Jeffrey/ Robert G. Eccles, 1989: Price, Authority, and Trust: From Ideal Types to Plural Forms. In: Annual Review of Sociology 15, 97-118.

Bräunling, Gerhard, 1983: Forschungs- und Technologiepolitik. In: Manfred G. Schmidt (Hrsg.), Westliche Industriegesellschaften. München: Piper, 106-111.

Brockhoff, Klaus, 1988: Forschung und Entwicklung: Planung und Kontrolle. München: Oldenbourg.

Bruder, Wolfgang (Hrsg.), 1986: Forschungs- und Technologiepolitik in der Bundesrepublik Deutschland. Opladen: Westdeutscher Verlag.

Bruder, Wolfgang/ Nicolai Dose, 1986: Forschungs- und Technologiepolitik in der Bundesrepublik. In: Wolfgang Bruder (Hrsg.), Forschungs- und Technologiepolitik in der Bundesrepublik Deutschland. Opladen: Westdeutscher Verlag, 11-75.

Bullinger, Hans-Jürg, 1990: Integrierte Produktentwicklung XV. Produktionsmittelhersteller und Zulieferer müssen weiterentwickeln. Eine Kooperation kann eigene $\mathrm{F}+\mathrm{E}-$ Kapazitäten für die Schlüsseltechnologien freimachen. In: Handelsblatt, 06.11.90, 20.

Cohen, Wesley M./ Daniel A. Levinthal, 1990: Absorptive Capacity: A New Perspective on Learning and Innovation. In: Administrative Science Quarterly $35,128-152$.

Danielmeyer, Hans-Günther, 1990: Spielraum für schöpferische Aktivitäten ist gefährlich. In: VDI-Nachrichten, 16.03.1990, 16.

Dörrenbächer, Christoph/ Michael Wortmann, 1991: The Internationalization of Corporate Research and Development. In: Intereconomics (May/ June), 139-144.

EIRMA (European Industrial Research Management Association), 1989: Cooperative R\&D in Industry. Working Group Reports 38. Paris: EIRMA. 
Esser, Josef, 1987: Does Industrial Policy Matter? Zum Einfluß industriepolitischer Konzepte auf die Technikentwicklung. In: Gerd Fleischmann/ Josef Esser (Hrsg.), Technikentwicklung als sozialer Prozeß. Frankfurt a. M.: Gesellschaft zur Förderung arbeitsorientierter Forschung und Bildung e.V., 123-135.

Esser, Josef, 1990: Does Industrial Policy Matter? Zur Rolle der Bundesländer in der Forschungs- und Technologiepolitik der Bundesrepublik Deutschland. In: Joachim Hirsch et al., Modernisierungspolitik heute. Die Deregulationspolitiken von Regierungen und Parteien. Frankfurt: Materialis, 57-71.

Fuhrmann, Frank Uwe/ Werner Väth, 1990: Staatliche Technologiepolitik nach der "Wende". In: WSI Mitteilungen 10, 622-629.

Hack, Lothar, 1988: Vor Vollendung der Tatsachen. Die Rolle von Wissenschaft und Technologie in der dritten Phase der industriellen Revolution. Frankfurt: Fischer.

Hack, Lothar, 1990: Industrieforschung - Vernetzung von globalen und lokalen Formen der Forschungs- und Technologiepolitik. In: WSI Mitteilungen $10,641-650$.

Häusler, Jürgen, 1989: Industrieforschung in der Forschungslandschaft der Bundesrepublik: ein Datenbericht. MPIFG Discussion Paper 89/1. Köln: MPI für Gesellschaftsforschung.

Häusler, Jürgen, 1990: Zur Gegenwart der Fabrik der Zukunft: Forschungsaktivitäten im bundesdeutschen Maschinenbau. MPIFG Discussion Paper 90/1. Köln: MPI für Gesellschaftsforschung.

Häusler, Jürgen, 1991: Adapting to an Uncertain Environment: R\&D in the West German Machinery Industry. In: Huib Ernste/ Verena Meier (Hrsg.), Regional Policy and Contemporary Industrial Response. Extending Flexible Specialisation. London: Belhaven (im Erscheinen).

Hartwich, Hans-Hermann (Hrsg.), 1986: Politik und die Macht der Technik. Opladen: Westdeutscher Verlag.

Jungnickel, Rolf et al., 1977: Einfluß multinationaler Unternehmen auf Außenwirtschaft und Branchenstruktur der Bundesrepublik Deutschland. Hamburg: Weltarchiv.

Kircher, Herbert, 1989: Der FuE-Manager muß die Grenzen einer Technologie erkennen und rechtzeitig die Verfahrens- oder Produktstrategie ändern. In: Handelsblatt, 24.10.89, 27.

Knorr-Cetina, Karin, 1984: Die Fabrikation von Erkenntnis. Zur Anthropologie der Naturwissenschaft. Frankfurt a. M.: Suhrkamp.

Kornhauser, William, 1962: Scientists in Industry. Berkeley: University of California Press.

Krohn, Wolfgang/ Günter Küppers, 1987: Die Selbstorganisation der Wissenschaft. Bielefeld: Kleine Verlag.

Küpper, Willi/ Günther Ortmann, 1986: Mikropolitik in Organisationen. In: Die Betriebswirtschaft 46(5), 590-602. 
Küpper, Willi/ Günter Ortmann (Hrsg.), 1988: Mikropolitik, Rationalität, Macht und Spiele in Organisationen. Opladen: Westdeutscher Verlag.

La Porte, Todd R., 1965: Conditions of Strain and Accomodation in Industrial Research Organizations. In: Administrative Science Quarterly 10, 21-38.

Lehner, Franz, 1986: Strukturen und Strategien der Technologiepolitik. In: Hans-Hermann Hartwich (Hrsg.), Politik und die Macht der Technik. Opladen: Westdeutscher Verlag, 242-263.

Luhmann, Niklas, 1990: Ökologische Kommunikation. Kann die moderne Gesellschaft sich auf ökologische Gefährdungen einstellen?, 3. Auflage. Opladen: Westdeutscher Verlag.

March, James G., 1988 (erstmals 1962): The Business Firm as a Political Coalition. In: James G. March, Decisions and Organizations. Oxford: Blackwell, 101-115.

Mayntz, Renate, 1985: Forschungsmanagement - Steuerungsversuche zwischen Scylla und Charybdis. Probleme der Organisation und Leitung von hochschulfreien, öffentlich finanzierten Forschungsinstituten. Opladen: Westdeutscher Verlag.

Narr, Wolf-Dieter/ Claus Offe, 1976: Was heißt hier Strukturpolitik? Neokorporativismus als Rettung aus der Krise? In: Technologie und Politik, Bd. 6/1976. Reinbek bei Hamburg: Rowohlt, 5-26.

OECD, 1991: Main Science and Technology Indicators. Paris: OECD.

Pausenberger, Ehrenfried, 1982: Technologiepolitik internationaler Unternehmen. Eine empirische Untersuchung über Forschung und Entwicklung, Technologietransfer und Technologieanpassung in internationalen Unternehmen. In: Zeitschrift für betriebswirtschaftliche Forschung, 1025-1054.

Pay, Diane de, 1989: Die Organisation von Innovationen. Ein transaktionskostentheoretischer Ansatz. Wiesbaden: Gabler.

Powell, Walter W., 1987: Hybrid organizational arrangements: new form or transitional development. In: California Management Review (Fall), 67-87.

Rammert, Werner, 1988: Das Innovationsdilemma: Technikentwicklung im Unternehmen. Opladen: Westdeutscher Verlag.

Rensen, Enno van, 1989: Industrieforschung - Innovation im Zusammenspiel von Grundlagenforschung und Unternehmenszielen. In: Konstanzer Blätter für Hochschulfragen 26(1-2), 57-65.

Ronge, Volker, 1977: Forschungspolitik als Strukturpolitik. München: Piper.

Ronge, Volker, 1986: Instrumentelles Staatsverständnis und die Rationalität von Macht, Markt und Technik. In: Hans-Hermann Hartwich (Hrsg.), Politik und die Macht der Technik. Opladen: Westdeutscher Verlag, 84-102.

Rosenberg, Nathan, 1990: Why do firms do basic research (with their own money)? In: Research Policy 19(2), 165-174. 
Rotering, Christian, 1990: Forschungs- und Entwicklungskooperationen zwischen Unternehmen - Eine empirische Analyse. Stuttgart: Poeschel.

Simonis, Georg, 1989: Technikinnovation im ökonomischen Konkurrenzsystem. In: Ulrich von Alemann/ Herbert Schatz/ Georg Simonis (Hrsg.), Gesellschaft. Technik. Politik. Perspektiven der Technikgesellschaft. Opladen: Leske + Budrich, 37-74.

Stucke, Andreas, 1991: Das Forschungsministerium des Bundes. Entstehung, Entwicklung und Steuerungsprobleme. Dissertation, Universität Bielefeld.

SV (Gemeinnützige Gesellschaft für Wissenschaftsstatistik $\mathrm{mbH}$ ), 1990: Forschung und Entwicklung in der Wirtschaft 1987 - mit ersten Daten 1989. Essen: SV.

Tulder, Rob van/ Junne, Gerd, 1988: European Multinationals in Core Technologies. Chichester: Wiley.

Väth, Werner, 1984: Konservative Modernisierungspolitik - ein Widerspruch in sich? Zur Neuausrichtung der Forschungs- und Technologiepolitik der Bundesregierung. In: PROKLA 56(3), 83-103.

Weinberg, Alvin M., 1970: Probleme der Großforschung. Frankfurt a. M.: Suhrkamp.

Weule, Hartmut, 1991: Aus weniger jetzt mehr machen. HighTech-Interview. In: HighTech (April), 84-86.

Wollmann, Hellmut, 1989: Entwicklungslinien der Technologiepolitik in Deutschland. Bestimmungsfaktoren, Zielsetzungen und politische Zuständigkeiten im Wandel. In: Jochen Hucke/ Hellmut Wollmann (Hrsg.), Dezentrale Technologiepolitik? Technikförderung durch Bundesländer und Kommunen. Basel: Birkhäuser, 35-75.

Zörgiebel, Wilhelm W., 1983: Technologie in der Wettbewerbsstrategie. Strategische Auswirkungen technologischer Entscheidungen untersucht am Beispiel der Werkzeugmaschinenindustrie. Berlin: Schmidt.

Zündorf, Lutz/ Manfred Grunt, 1982: Innovation in der Industrie. Organisationsstrukturen und Entscheidungsprozesse betrieblicher Forschung und Entwicklung. Frankfurt a. M.: Campus.

Zweipfennig, Heribert, 1991: Der Zusammenhang zwischen der Organisation und der Produktivität industrieller Forschung und Entwicklung. Göttingen: WiRe. 